\section{Sengstaken Balloon Tamponade for Life-Threatening Pouch Hemorrhage after Laparoscopic Gastric Banding}

Laparoscopic gastric banding is advocated as a minimally invasive procedure with a low risk profile and high efficacy in the treatment of morbid obesity.

A 34-year-old woman with a body mass index of 48 underwent a laparoscopic gastric banding operation (Swedish adjustable gastric banding, SAGB). The operative technique was performed according to the described and standardized technique [1-3]. A gastric tube was placed in the stomach during retrogastric tunnelling with the goldfinger (Obtech Medical AG, is a smooth tool recommended for dissecting and pulling of the band) and removed after closure of the band and before creation of the pouch. The small pouch was created and secured with five anterior gastrogastric nonabsorbable sutures. The intraoperative and immediate postoperative follow-up was uneventful, but during the first postoperative night the patient developed severe melena and hematemesis. At emergency endoscopy, brisk profound bleeding from the pouch was found with no additional bleeding source. Despite aggressive endoscopic sclerotherapy with injection of, in total, $32 \mathrm{ml}$ of $1 \%$ Aethoxysclerol (Kreussler Pharma, Wiesbaden-Biebrich, Germany) over two occasions, hemostasis could not be achieved. Shock necessitated intensive care observation and the transfusion of 9 units of packed red cells. A Sengstaken-Blakemore tube was introduced (Figure 1) and blocked. The esophageal balloon was filled with $40 \mathrm{ml}$ of water and the stomach balloon was inflated with $120 \mathrm{ml}$ of air. After placement of the tube, immediate hemostasis was achieved. No further signs of bleeding occurred and a gastroscopy 1 day later confirmed hemostasis. The patient was subsequently discharged on the fifth postoperative day. At 1- and 2-month follow-up the patient had no complaints and had a total weight loss of $23 \mathrm{~kg}$. All laboratory values were in the normal range.

We hypothesize that the bleeding was related either to the removal of the gastric tube by the anesthetist while applying suction to the tube or to one of the pouch sutures. Control of such bleeding using an endoscopic approach is nearly impossible because the pouch is too small to allow direct vision. In such a situation, we

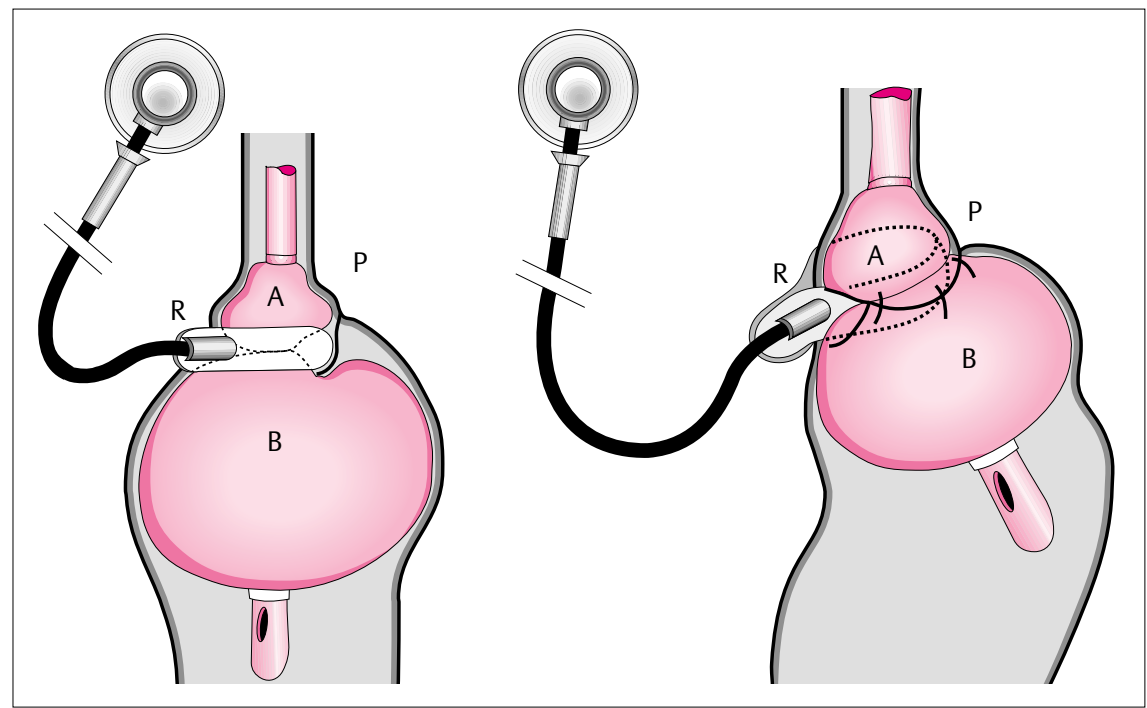

Figure 1 The Sengstaken-Blakemore tube. A, esophageal balloon; B, gastric balloon; P, pouch; R, adjustable band

recommend the use of the SengstakenBlakemore tube with the method described above.

\section{H. Nehoda, H. Weiß, B. Labeck,}

M. Lanthaler, K. Hourmount,

M. Oberwalder, F. Aigner

Dept. of General Surgery,

University Hospital of Innsbruck,

Innsbruck, Austria

\section{References}

${ }^{1}$ Belachew M, Legrand MJ, Vincent V, et al. Laparoscopic adjustable gastric banding. World J Surg 1998; 22: 955-963

${ }^{2}$ Forsell P, Hellers G. The Swedish adjustable gastric banding (SAGB) for morbid obesity - nine-year experience and four year follow up of patients operated with a new adjustable band. Obes Surg 1997; 7: $345-351$

${ }^{3}$ Klaiber CH, Metzger A, Forsell P. Laparoskopisches gastric banding. Chirurg 2000; 71: 146-151
Corresponding Author

H. Nehoda, M.D.

Dept. of General Surgery

University Hospital of Innsbruck

Annichstrasse 35

6020 Innsbruck

Austria

Fax: +43-512-504-2605

E-mail: Hermann.Nehoda@uibk.ac.at 